\title{
The study of the process of physic-chemical destruction of coal by the method of physical modeling
}

\author{
Serhii Makeiev ${ }^{1, *}$, Serhii Andreiev ${ }^{1}$, and Hennadii Ryzhov ${ }^{1}$ \\ ${ }^{1}$ Institute of Geotechnical Mechanics named by N. Poljakov of National Academy of Sciences of \\ Ukraine, 49005, Dnipro, Simferopolska Str., 2a, Ukraine
}

\begin{abstract}
One of the possible ways of physic-chemical destruction (PCD) of coal was considered. It is established that condition of the realization of a principle possibility of spontaneous dispersion of coal is to reduce its surface energy by $40-60 \mathrm{~J} / \mathrm{m}^{2}$. Laboratory experiments to study the PCD of coal in different liquids were conducted. According to the results, kinetic curves of swelling coal in them transition D-G stamps is presents. It was established that the most intensive physical and chemical destruction of the coal to a particle size from tenths to tens of millimeters occurs in solutions of methylamine and hydrochloric acid. The most severe swelling of the coal was noted in those environment as well. The intensity of swelling is not significantly associated with the characteristics of wetting. The influence of various effects on the PCD parameters of coal was studied by physical simulation. The total time the chemical destruction of coal on the treated area was calculated. It was proposed method PCD for coals occurring in the clay rocks of various stages of catagenesis.
\end{abstract}

\section{Introduction}

Physic-chemical influence methods on the coal seams and host rock with the aim of improving the safety and efficiency of mining operations was widely spread in the development of coal deposits [1]. Various chemical solutions aimed at strengthening rocks, and on their softening are used as working fluids [2]. At the same time, the mechanisms of interaction of coals with these substances still were no insufficiently studied, which greatly hinders the search for new environments and the development of methods of physicchemical effects on rock mass array. Therefore, the study and understanding of these mechanisms, and further their use as a control action (physical-chemical influence on the rock mass massif with the aim of strengthening and weakening or destruction) with the use of different chemical solutions could make a significant contribution to the development of the coal industry and mining industry as a whole.

The prospect of physico-chemical destruction of the coal due to its colloidal-chemical properties, namely, strains and stresses of swelling, which can reach tens of megapascals. Therefore, the search for and study of different chemical solutions that are able to cause

*Corresponding author: smakeev@ukr.net 
these phenomena in coal, is quite a topical area of research.

\section{Methods}

One of the possible ways of physic-chemical destruction of coal is the use of spontaneous dispersion, based on the Rebinder effect. The process of spontaneous dispersion are divided into two different in their nature phenomena: a true spontaneous dispersion of particles of colloidal dimensions with the formation of the thermodynamically equilibrium lyophilic colloidal systems and dispersion quasimonopolies resulting in the formation of a relatively stable, prone to collective recrystallization, and coarse systems [3].

The thermodynamic condition for the occurrence of true spontaneous dispersion is the decrease in free energy of the system due to compensation of the increase of free surface energy $\sigma_{m}$ in the formation of particles with well-developed surface. Compensation is caused by the increase of entropy of the system as a result of the involvement of the produced particles in Brownian motion. True spontaneous dispersion of bulk phase on the particle size $\delta$ is possible if the value of the specific free surface energy of the interface of a solid matter with a surface-active medium will satisfy the condition Rehbinder and Shchukin $[3,4]$ :

$$
\sigma_{12} \leq \sigma_{m}=\gamma k T / \delta^{2}
$$

where $\gamma$-a numerical multiplier of about $10 ; k$ - the Boltzmann constant, $\mathrm{J} / \mathrm{K} ; T-$ the absolute temperature, $\mathrm{K}$.

Let's rate the value $\sigma_{m}$. If the coal structure with point contacts and effective size of about $3 \cdot 10^{-8} \mathrm{~cm}$ is imagined, then $\sigma_{m}$ will be approximately $10^{-1} \mathrm{~J} / \mathrm{m}^{2}$. If we take the upper limit of the particle size in true spontaneous dispersion equal to the size limit of particles that can be involved in Brownian motion $\delta=5 \cdot 10^{-4} \mathrm{~cm}$, then the calculations of equation (1) give $\sigma_{m} \approx 10^{-9} \mathrm{~J} / \mathrm{m}^{2}$. Thus, thermodynamic calculations show, that the possibility of a genuine spontaneous dispersion, it is necessary to reduce the surface energy to $10^{-1}-10^{9} \mathrm{~J} / \mathrm{m}^{2}$.

Kinetic analysis of the process of spontaneous dispersion of solids in the medium of surfactants is given in paper [5]. Surface-active environment, that is similar in molecular nature to a solid, reduces its surface tension and already at low tensile stresses, brittle rupture is observed. The surface energy of a solid body can be reduced so, that not condensed, but colloidal condition becomes more thermodynamically stable, and the body begins spontaneously, without external stress get dispersed apart.

The kinetics of this type of fracture is determined by the presence of structurally weakened the boundaries between micro areas and the internal stress of the second kind on those borders. The only decrease the surface tension is not enough so that the gap occurred, it is necessary to have tensile stress in the top of the cracks. According to the paper [5], spontaneous dispersion of solids is possible, if the total tensile stress at the top of the microcracks exceeds the safe stress in this environment. In turn, the total voltage is the sum of existing on the border of the blocks of internal stresses of the second kind and the incremental stresses due to pressure of the adsorbed layer.

Kinetic calculations based on the mechanism of microcracks growth [5], show that

$$
\sigma_{m}=k_{1} \sigma_{0} /\left(2+k_{1}\right)
$$

where $\sigma_{0}$ - the specific surface energy of the body in surface-inactive medium; $k_{1}-$ coefficient determined by the geometry of the microcracks and equal to $0.3-2.0$. For other coals $\sigma_{0} \approx 10^{-2} \mathrm{~J} / \mathrm{m}^{2}[6], \sigma_{m} \approx 13-60 \mathrm{~J} / \mathrm{m}^{2}$. 
Thus, the results of a thermodynamic and kinetic analysis show that the surface energy of the coal must be reduced by 40 to $60 \mathrm{~J} / \mathrm{m}^{2}$ for the realization of the principal possibility of spontaneous dispersion. Such a reduction cannot be achieved using only the Rebinder effect. Thus, for aqueous solutions with a surface tension of the order of $10^{-1} \mathrm{~J} / \mathrm{m}^{2}$ and contact angle of wetting, close to zero, reducing the surface energy during wetting is only $10^{-1} \mathrm{~J} / \mathrm{m}^{2}$, i.e. three orders of magnitude smaller than required.

The quasimonopolies dispersion through the cracks formation of destruction under the combined action of the strongly adsorptive media and internal tensile stresses is practically impossible, mainly due to the inability of a significant adsorption reduces surface energy and low level of residual internal stresses in the coal.

\section{Results and discussion}

As it is shown by experiments, the manifestation of the effect of physic-chemical destruction of coals by using aqueous solutions of a number of known surface-active substances is possible only for a very the prepared carbons, which have a degree of natural disturbance is so great that they cannot be considered solid.

The kinetic curves of swelling of the coal transition D-G brands in various liquids are shown in Figure 1. The characteristics of coal wetting with these solutions are shown in Table 1.

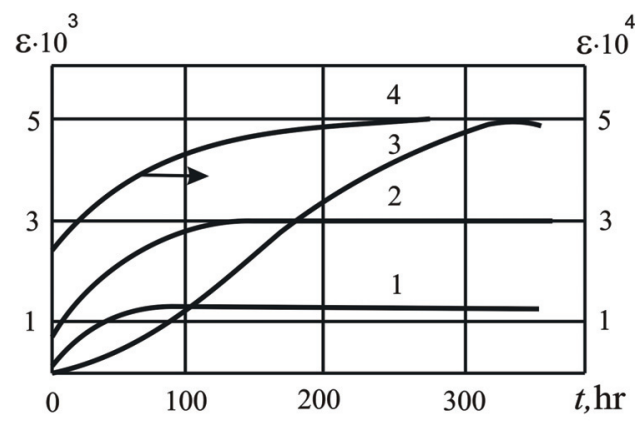

$$
\varepsilon \cdot 10^{3}
$$

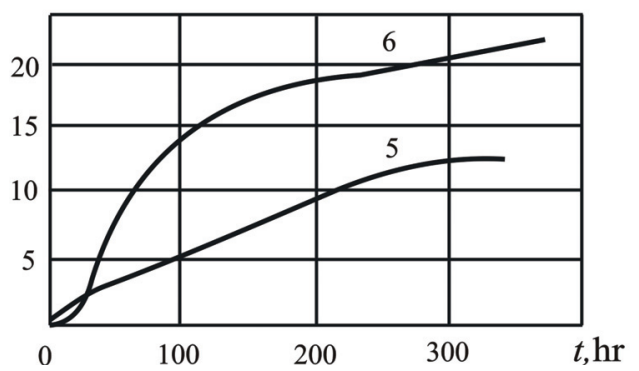

Fig. 1. The dependence of the relative linear deformations of the coal swelling $\varepsilon$ in various fluids from time: 1 - water; 2 - $25 \%$ solution of ammonia; 3 - acetone; 4 - isopropanol (right axis); 5 $25 \%$ solution of methylamine; $6-36 \%$ hydrochloric acid.

As it is shown in Table 1, the most intense physical and chemical destruction of the coal to a particle size from tenths to tens of millimeters occurs in solutions of methylamine and hydrochloric acid. The strong swelling of the coal is noted in them as well. The intensity of swelling is not significantly associated with the characteristics of wetting. So, water has more wetting energy, than a solution of methylamine (see Table 1), and coal swells in it 
order of magnitude weaker. Liquids with almost the same wetting energy (isopropanol and acetone) differ the deformation of swelling almost two orders of magnitude.

Table 1. Characteristics of coal wetting with various liquids.

\begin{tabular}{|l|c|c|c|}
\hline \multicolumn{1}{|c|}{ Liquid } & $\begin{array}{c}\text { Surface tension, } \\
\sigma \cdot 10^{3} \mathrm{~J} / \mathrm{m}^{2}\end{array}$ & $\begin{array}{c}\text { Edge angle, } \\
\mathrm{deg}\end{array}$ & $\begin{array}{c}\text { The wetting energy, } \\
A \cdot 10^{3} \mathrm{~J} / \mathrm{m}^{2}\end{array}$ \\
\hline Isopropanol & 20 & 0 & 20 \\
Water & 72 & 65 & 41 \\
$25 \%$ ammonia & 50 & 52 & 31 \\
Acetone & 23 & 0 & 23 \\
$36 \%$ hydrochloric acid & 70 & 27 & 62 \\
$25 \%$ methylamine & 42 & 47 & 29 \\
\hline
\end{tabular}

The investigation of influence of different effects on the PCD parameters of coal was carried out by the method of physical modeling. The conditions and criteria of similarity for modelling these processes, which should describe both hydrodynamic and mechanical parameters is defined further. The movement of reagents that cause PCD coal in its fractured-porous structure, is described by the following hydrodynamic criteria [7]: Reynolds, characterizes the ratio of inertia forces to viscosity forces; Bond, characterizes the ratio of gravity forces to the capillary; Weber, characterizes the ratio of inertial forces movement of the reagent to the capillary. In the experiment the values of the criterion Reynolds had the value of about $10^{-1}-10^{0}$.

The coal, which was used in model experiments, is real object of PCD, so the equality criteria of the Bond and Weber for the processes of PCD is respected inside the blocks of coal in the array and models.

The value of the dimensionless complex was taken to achieve similarity parameters of mechanical loading as a dimensionless similarity criterion, as it is normally used for such purposes:

$$
\frac{\sigma^{2}}{E A_{d}},
$$

where $\sigma$ - stress, Pa; $E$ - the modulus of elasticity destructible environment, $\mathrm{Pa} ; A_{d}-$ work of coal destruction, J. The values of $E$ and of $A_{d}$ for the model and the coal seam are accepted in the first approximation the same.

If the energy of the explosion is used as an impulse mechanical stress, initiating the process of PCD, then the well-known criterion of geometric similarity will be:

$$
\frac{L}{\sqrt[3]{W}},
$$

where $L$ - the characteristic size, $\mathrm{m} ; W$ - the energy of the explosion, $\mathrm{J}$.

Preliminary softening of coal under the action of any solution significantly reduces the size of pieces by crushing. If the destruction of low-strength coal produced mainly under the influence of the expanding gaseous products of the explosion, then the shock wave passes for a time that longer than the time of formation of plastic zone of cracks fracture.

The low acoustic stiffness weakening coal contributes to the effects of this damage mechanism. In this case, the average lump volume by crushing will be determined as [8]

$$
J=\frac{E e}{\sigma_{1}^{2}} f\left(\frac{\sigma_{1}}{\sigma_{0}}\right),
$$


where $e$ - energy required to form unit surface cracks, $\mathrm{J} ; \sigma_{1}-$ the maximum stress in the blast wave, $\mathrm{Pa} ; \sigma_{0}-$ initial compressive stress in the coal, $\mathrm{Pa}$.

The softening of coal reduces its modulus of elasticity in two to three times, and reduces the energy required per unit of surface cracks in three-four times. Thus, softening of coal for the same explosive charge leads to a decrease in the size of pieces after fragmentation 612 times, facilitating the excavation of coal at a constant $\sigma_{1}$ and $\sigma_{0}$.

The experiments were carried out in the apparatus for uniaxial compression of a flat sample with a size $12 \times 8 \times 2 \mathrm{~cm}$. The hole for the discharge of a $25 \%$ solution of methylamine was drilled right in the center along the length of the sample to a depth of $8 \mathrm{~cm}$ and a diameter of $0.5 \mathrm{~cm}$. From 3.2 till 4.7 liter of solution was injected only for 230 280 hours.

The averaged results of the experiments are given in Table 2, which shows that over time the swelling slows down, this reduces the number of microcracks formed recorded by the acoustic emission method. Through 230-280 hours discharge pressure in the sample reduced drastically, and the filtration speed increased repeatedly due to the formation therein of macro cracks detection.

Table 2. The PCD parameters of the coal in the clamped flat sample.

\begin{tabular}{|c|c|c|}
\hline $\begin{array}{c}\text { The time of } \\
\text { discharge, } \mathrm{hr}\end{array}$ & $\begin{array}{c}\text { Movement of the } \\
\text { sample end face, } \mathrm{mm}\end{array}$ & $\begin{array}{c}\text { The count rate of acoustic } \\
\text { emission, impulse/s }\end{array}$ \\
\hline 5 & 0.18 & 24 \\
25 & 0.44 & 18 \\
50 & 0.84 & 16 \\
75 & 1.07 & 27 \\
100 & 1.59 & 23 \\
150 & 2.08 & 19 \\
200 & 2.14 & 6 \\
225 & 2.16 & $0-2$ \\
\hline
\end{tabular}

After removing the sample cracks was recorded predominantly perpendicular to the injection hole. Samples lost integrity. The peeling of coal with the formation of more fines was on the borders of macro cracks detection. The maximum size of the separateness is the order of the sample thickness, the smallest of the formed particles is dust dispersion (Table 3).

Table 3. Fractional composition of destroyed coal.

\begin{tabular}{|c|c|c|}
\hline The fractions size, $\mathrm{mm}$ & The average fractions size, $\mathrm{mm}$ & Composition, $\%$ \\
\hline $20-50$ & 35 & 71.4 \\
$10-20$ & 15 & 11.8 \\
$7-10$ & 8.5 & 2.1 \\
$3-7$ & 5 & 6.5 \\
$1-3$ & 2 & 4.2 \\
$0.5-1$ & 0.75 & 2.9 \\
$0.25-0.5$ & 0.375 & 0.8 \\
$0-0.25$ & 0.125 & 0.3 \\
\hline
\end{tabular}

Physical and chemical destruction of the coal can be achieved by forcing the layers of oxygen-containing oxidizers as well, and then the aqueous solutions with an alkaline reaction. The process of the PCD in this case is follows. Through wells in the coal seam oxygen-containing oxidizing agents such as nitric acid, solutions of potassium permanganate, chromic and sulfuric acid, ozone, hydrogen peroxide, air, etc., or such oxidizing agents to initiate the oxidation process as pyridine, benzoyl peroxide, cumene 
hydroperoxide, etc. are injected. During the injection of coal seam of oxygen-containing oxidizers or oxidizers to initiate the oxidation process of coal, the oxidation of organic part of the latter occur with the formation of oxygen-containing groups (peroxides, coal-acids complexes, a hydro carboxyl, carbonyl), as well as various organic acids, primarily humic $[9,10]$. The oxidation of coal occurs at the boundaries of macro - and micro-cracks and macropores that divide the coal into blocks, including oxidized unbroken contact blocks, giving the coal seam a holistic, coherent character. Then aqueous solutions with alkaline reaction, such as solutions of potassium hydroxide, liquid glass, etc. are injected in the coal seam. Alkaline solutions dissolve the formed humic and other acids and obtained by oxidation of coal oxygen groups contribute to the solubility of humic acids in these solutions [10].

Dissolution at the contacts and the block boundaries of acidic products of coal oxidation causes the loss of the last integrity, transfer in non-cohesive in nature, i.e. chemical degradation. This purely chemical destruction is practically independent from the forces of rock pressure and occurs throughout the volume of the treated area of the massif (Fig. 2).

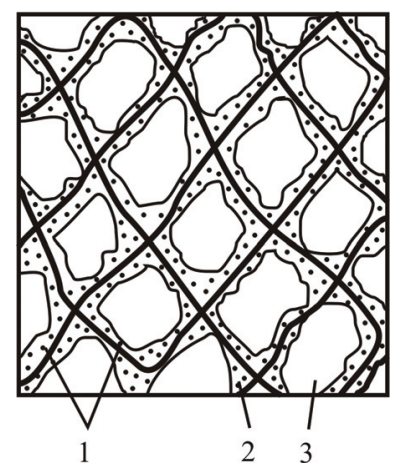

Fig. 2. The coal structure after its physic-chemical destruction: 1 - cracks, which divide the coal into blocks; 2 - coal, which dispersed (or dissolved) at the surface of cracked; 3 - blocks unchanged, one piece of coal.

The implementation of this method practically does not lead to deterioration in the quality of extracted coal due to oxidation. Because of the developed coal seams are usually full of methane, and it dramatically reduces the ability of the coal to oxidation, so the latter will take place on the contacts and the boundaries of the coal blocks, which are gas-free by injection of oxygen-containing oxidants in the first place, while the bulk of the coal remains not gas-free and unoxidized. The products of oxidation of the coal are dissolved and carried out of the reservoir of the filtration flow by injection of aqueous solutions with alkaline reaction.

It is expedient to use oxidizing agents that cause embrittlement (antipathetical) coal: oxygen, ozone, etc. Thus the process of mechanical destruction of coal including on contact blocks becomes easy.

Computational example of this method implementation is presented below. The wells are drilled, that stripped the coal seam, and the injection of oxygen-containing oxidizing agent for example an aqueous solution of chromic acid is conducted through them. The radius of one well aqueous solution of chromic acid processing is $20 \mathrm{~m}$. Filtered by macrocracks and macropores, acid causes oxidation of borders and contacts of coal blocks to form various products, mainly humic acids.

The oxidation time of the coal layer of $1 \mathrm{~mm}$ thick, which occurs in the diffusion mode is calculated below.

The famous analogy in the mathematical description of thermal and diffusion processes is used to calculate the time of penetration of the oxidation front $\tau_{1}$ according to the 
following formula [7]:

$$
\tau_{1}=\Delta \sqrt{\frac{\tau_{0}}{D}},
$$

where $\Delta-$ the thickness of the oxidized layer, $10^{-3} \mathrm{~m} ; \tau_{0}-$ the period of relaxation for the process of mass transfer, has the order of $10^{2} \mathrm{~s} ; D$ - the diffusion coefficient of oxidizer in a solid body, that has the order of $10^{-12} \mathrm{~m}^{2} / \mathrm{s}$.

The calculation gives a value of $\tau_{1}$, that equal to $10^{4} \mathrm{~s}$, or about 3 hours.

Then a solution of alkaline reaction, such as a solution of potassium hydroxide is injected in the coal seam. The alkaline solution dissolves the formed humic and other acids, while coal loses its integrity, coherence, chemically separating into blocks.

The dissolution time of layer thickness $1 \mathrm{~mm}$ is calculated below.

The mass of the oxidizing layer with a thickness of $1 \mathrm{~mm}$ per $1 \mathrm{~cm}^{2}$ of the coal is $M=0.15 \mathrm{~g} / \mathrm{cm}^{2}$, assuming a coal density and the density of its oxidized products approximately the same and equal to $1.5 \mathrm{~g} / \mathrm{cm}^{3}$. The dissolution time of layer $\tau_{2}$ are defined as:

$$
\tau_{2}=\frac{M}{\xi}
$$

where $M$ - mass of the oxidized layer, $0.15 \mathrm{~g} / \mathrm{cm}^{2} ; \xi$ - stream solution, $\mathrm{g} /\left(\mathrm{cm}^{2} \cdot \mathrm{s}\right)$.

Since the filtration of alkaline solutions in the cracks of coal occurs in laminar flow to determine the flow of dissolution the equation of Levich [11] is used for laminar flow in the pipe in the area of diffusion of the input area:

$$
\xi=0.67 C_{0} D\left(\frac{U_{0}}{D R x}\right)^{\frac{1}{3}},
$$

where $C_{0}$ - the concentration of a saturated solution of humic acid in alkali, $0.2 \mathrm{~g} / \mathrm{cm}^{3}$; $D$ - the diffusion coefficient of humic acid in alkali, $10^{4} \mathrm{~cm}^{2} / \mathrm{s} ; U_{0}-$ the speed of the alkaline solution in a crack of $0.1 \mathrm{~cm} / \mathrm{s} ; R$ - the equivalent radius of the cracks, $10^{-2} \mathrm{~cm} ; x-$ the average distance from the borehole, that will be taken equal to half the radius of the treated area $10^{3} \mathrm{~cm}$.

The calculation yields a value of $\xi=6 \cdot 10^{-5} \mathrm{~g} /\left(\mathrm{cm}^{2} \cdot \mathrm{s}\right)$, and accordingly $\tau_{2}=2.5 \cdot 10^{3} \mathrm{~s}$, or about 1 hour.

Thus, the total time of the coal chemical destruction on the treated area will be $\tau=\tau_{1}+$ $\tau_{2}=3+1=4$ hours.

The use of this process is possibly on the coals occurring in argillaceous rocks as well. If shale is quite high stages of catagenesis, and is not prone to significant soaking, then the contact failure of coal - rock can hold the impact of solutions, chemically destroying such formations, for example 2-5\% solution of urea. In this case, the coal will "slide" on the substrate of sodden rocks. By specifying certain of urea concentration and time of exposure, it is possible to obtain a thin film of sodden rocks, that adjacent to the reservoir, without disintegration or breaking of rock mass of the roof.

If clay rocks are low stage of catagenesis (mudstones, siltstones), then it is possible to obtain a thin film sodden rocks without soaking deep into the underlying array, pumping solutions, inhibitors of hydration of clay minerals, for example 2-5\% solution ferrochromolignosulfonat. 


\section{Conclusions}

Thus, the results of a thermodynamic and kinetic analysis show that the implementation of the principle possibility of spontaneous dispersion of coal, its surface energy must be reduced from 40 till $60 \mathrm{~J} / \mathrm{m}^{2}$. Such a reduction cannot be achieved using only the Rebinder effect. Thus, for aqueous solutions with a surface tension of the order of $10^{-1} \mathrm{~J} / \mathrm{m}^{2}$ and edge angle of wetting, close to zero, reducing the surface energy during wetting is only $10^{-1} \mathrm{~J} / \mathrm{m}^{2}$, i.e. three orders of magnitude smaller than it is required. The physic-chemical method of destruction of various liquids based on the action of the stresses of swelling, adsorption reduction of strength of the coal, chemical oxidation and subsequent leaching of the oxidation products at the border of the coal blocks was proposed and tested in the laboratory.

The physical simulation of the process physic-chemical destruction of the coal in stress state in terms of internal filtration, the total time the chemical destruction of coal on the treated area is calculated. The method PCD for coals occurring in the clay rocks of various stages of catagenesis is proposed.

\section{References}

1. Makeiev, S.Yu., Andreiev, S.Yu., Ryzhov, H.A. (2016). Reducing the outburst hazard of the massif by increasing the homogeneity of the fractured-block structure. Geotekhnicheskaya Mekhanika [Geotechnical mechanics], 127, 67-76

2. Bulat, A.F., Makeiev, S.Yu., Andreiev, S.Yu., Ryzhov, H.A., Gorska, T.P. (2015). Sposib upravlinnja stanom vugleporodnogo masivu. Patent No 97444, Ukraine

3. Shchukin, E.D., Goryunov, Yu.V., Pertsev, N.V. (1974). The dispersion of solids in the presence of adsorption-active media. Adhesion of melts. Kyiv: Naukova dumka, 15-19

4. Frolov, Yu.G. (1982). Course of colloid chemistry. Surface phenomena and disperse systems. Moskva: Khimiya

5. Brtenev, G.M., Yudina, I.V., Rebinder, P.A. (1958). The theory of spontaneous dispersion of solids. Colloid journal, 5, 655-664

6. Ivanov, B.M., Feyt, G.N., Yanovskaya, M.F. (1979). Mechanical and physico-chemical properties of outburst-hazardous coal seams. Moskva: Nauka

7. Oksanich, I.F., Mironov, P.S. (1982). Laws of rocks crushing by explosion and predicting the granulometric composition. Moskva: Nedra

8. Lykov, A.V. (1981). Heat and mass transfer. Moskva: Energiya

9. Kasatochkin, V.I., Larina, N.K. (1975). Structure and properties of natural coals. Moskva: Nedra

10. Kukharenko, G.A. (1960). Chemistry and Genesis of fossil coal. Moskva: Gosgortekhizdat

11. Levich, V.G. (1959). Physico-chemical hydrodynamics. Moskva: Fizmatgiz 